\title{
Study on Location Algorithms of Beamforming based on MVDR
}

\author{
Aidong Deng ${ }^{1, *}$, Hang Tong ${ }^{2}$, Jianeng Tang ${ }^{3}$, Hao Cao ${ }^{4}$, Kang Qin $^{1}$ and Xi Yan ${ }^{1}$ \\ ${ }^{1}$ National Engineering Research Center of Turbo-generator Vibration, Southeast University, Nanjing 210096, China \\ ${ }^{2}$ Huadian Electric Power Science Research Institute, Hangzhou 310030, China \\ ${ }^{3}$ College of Engineering, Huaqiao University, Quanzhou, Fujian 362021, China \\ ${ }^{4}$ Hunan Electric Power Corporation Research Institute, Changsha 410007, China
}

Received: 3 Apr. 2013, Revised: 7 Aug. 2013, Accepted: 8 Aug. 2013

Published online: 1 Nov. 2013

\begin{abstract}
Acoustic emission is an effective method of locating the rubbing fault. In order to solve the problem that satisfactory location accuracy is difficult to obtain because of the waveform distortion caused by signal propagation during the application of time delay estimation method in acoustic emission position estimation, beam-forming technique is applied to acoustic emission source location. Simulation studies have been made on the performance of near-field time-domain and frequency domain beam-forming in the location of rubbing acoustic emission source. The paper adopts the wideband signal minimum variance distortionless response (MVDR) location estimation method based on sub-band decomposition to avoid the problems of poor noise immunity and low resolution of traditional beam-forming. Decompose each group of array signals into a number of sub-band of equal length, conduct Fourier transformation on each sub-band to calculate the covariance matrix of each frequency component, get the two-dimensional joint distribution function of the MVDR output power of each sub-band with respect to the distance and azimuth angle, then synthesize the MVDR power of wideband signal, obtain the azimuth spectrum estimation of all frequency bands, and finally get the location of the acoustic source by the peak point. The experimental results show that this algorithm can accurately identify the rubbing fault location.
\end{abstract}

Keywords: Acoustic emission, beamforming, MVDR, rubbing, location.

\section{Introduction}

Rotating machinery rubbing fault diagnosis technique based on acoustic emission (AE) has attracted more and more attention from scholars in recent years. Acoustic emission technique can not only determine the occurrence of rotor rubbing fault, but also quickly find the rubbing location by $\mathrm{AE}$ source location technique which provides important information for the analysis of the fault cause and the troubleshooting. Sato [1] simulated the continuous rubbing state on a $350 \mathrm{MW}$ steam turbine unit, he pointed out that characteristic parameters got by detecting $\mathrm{AE}$ envelope signal can be used as generation indicators of rubbing. Suzuki [2] used wavelet transformation to seek event counts or event count rate of characteristic parameters of AE signal, he also used this method for rubbing fault detection of rotating machinery. Mba [3] studied the propagation of AE signal in large steam turbine generator unit by choosing the low pressure rotor of a $550 \mathrm{MW}$ steam turbine as the detection object.
Experiment results showed that AE signal waveform generated at any point of the turbine shaft can be detected at both ends of the shaft. Besides, through the rubbing location experiment, he pointed out that amplitude modulation of AE signal provides important information of interaction force of the rotor and the stator, which provides an effective method for rubbing location. Hall [4] presented a diagnosis of continuous rotor-stator rubbing based on the experiment on an operational $500 \mathrm{MW}$ turbine unit via high frequency $\mathrm{AE}$ measurement, and calculated the rubbing location by using the phase delay between adjacent AE modulations. Time delay estimation algorithm is generally adopted to calculate the time difference of arrival (TDOA) in acoustic emission location, and determine the location of sound emitting source according to the geometric structure of the sensor. Omologo [5] proposed a cross-power spectrum phase delay estimation algorithm, achieving precise location of point acoustic sources in

\footnotetext{
*Corresponding author e-mail: addseu@163.com
} 
three-dimensional space under different conditions of noise and echoes; Yegnanarayana [6] extracted short-term spectral characteristics by dividing two time series of homologous sound signals into several segments of equal length, implemented time delay estimation on each set of segments based on this and he got better location effect than that of generalized cross-correlation. Deng [7] proposed generalized cross correlation (GCC) time delay estimation of the best linear fraction Fourier transformation domain filtering based on the same fractional order factor and different fractional order factor. Furthermore, to solve the problem that the maximum related point can not be obtained due to waveform variation caused by frequency dispersion effect in the propagation of acoustic emission signal, he presented improved signal processing methods of segmented correlation multiplication processing and segmented correlation exponential transformation processing. Ciampa [8] proposed an algorithm based on wavelet analysis and a Newton-based optimization technique to identify the real-time acoustic emission location, this method overcame the drawbacks of the triangulation method in terms of estimating a priori group velocity and the need to find the best time-frequency technique for time-of-arrival determination. Dirk Aljets [9] used three sensors installed in a triangular array to establish the direction from the sensor array to the $\mathrm{AE}$ source by analyzing the arrival times of A0 component of the signal to the three sensors, the distance can also be evaluated by the separation of S0 and A0 mode at each sensor respectively. To sum up, these algorithms based on time delay estimation is to get the sample time delay by calculating the time domain cross-correlation of different sensor signals, and then calculate the spatial location of acoustic source according to acoustic signal propagation theory and array position. They require that the obtained time delay of sensors must be very accurate. However, these algorithms are largely restricted in rotor system structure. The reason is as follows: in rotor system, it is usually a length of non-continuous and non-single-medium complex propagation path from the rubbing source to the sensor, the rubbing motivated multi-modal AE wave has a serious signal distortion due to the influence of many effects such as boundary conditions, frequency dispersion and mode conversion, etc. in the propagation process. The map between the AE source and the AE signals received by the sensor is nonlinear, the signal reaching the sensor is a superposition of multimode waves with noise from different paths, and every channel sensor will collect poor correlation waveforms so that it is difficult to calculate the accurate arrival time difference [10]. Beam-forming is a signal processing technology measuring space radiation acoustic field information by sensor array $[11,12]$. The basic idea is that do the weighted sum processing on each element output of the array, and according to different optimization criteria, make the array output produce different responses to signals from different spatial directions so as to realize that the array beam points to the expected signal and the null points to the interfering signal. Beam-forming technology can not only estimate the acoustic source direction of arrival (DOA), but also achieve the precise location of near-field acoustic source. Compared with the traditional time difference location method, beam-forming method has many advantages such as little impact of the channel attenuation, convenient sensor placement, less sensors, lower sampling rate and simultaneous location of multiple acoustic sources. It has been widely used in military technology fields such as radar, propagations, electronic warfare, sonar as well as aerospace technologies. AE location technology based on beamforming also is also studied in recent years. For the damage of large structures in civil engineering, McLaskey [13] implemented nondestructive testing researches based on acoustic emission beamforming method, their study results showed that beamforming methos, compared with traditional methods, is more economical and practical because it is convenient for sensor placement, it can reduce the number of sensors to be used, it can reduce the synchronization requirements of arrival time in all channels and it can adopt a relatively lower sampling rate. He [14] conducted researches on acoustic emission signal propagation characteristics which proved the feasibility of beamforming in acoustic emission signal processing by the use of guided wave theory. Besides, he used near-field acoustic emission beamforming method to locate rotor-stator rubbing fault and successfully identified its location. For problems of poor noise immunity and low resolution of traditional beam-forming method, this paper adopts the minimum variance distortionless response (MVDR) [15,16,17] beam-forming method to improve the location accuracy and enhance the noise interference suppression performance of rubbing acoustic emission source location. At the same time, according to the wideband characteristics of rubbing acoustic emission signals, this paper adopts wideband near-field signal MVDR beam-forming algorithm based on sub-band decomposition. This paper has analyzed the performance of traditional beam-forming method and wideband near-field signal MVDR algorithm based on sub-band decomposition in rubbing acoustic emission source location through simulation and experiments.

\section{Traditional near-field beam-forming}

Beam-forming method can be classified into far-field and near-field according to the distance between the acoustic source and the array. The far-field beam-forming method bases on the fact that the signal source is located in infinity, the incident signal can be considered a plane wave, and there is no energy loss of the signal in the propagation process. And the near-field beam-forming method bases on the fact that the acoustic source is a point source which is the source point of divergent propagation of the signal to the space around, there is an 
acoustic energy attenuation in the propagation process, and the longer the distance is, the greater the attenuation will be. Attenuation of acoustic emission is very obvious in the propagation process, so sensors shall be placed near the acoustic emission source as much as possible, all these make it difficult to use the assumption of far-field, thus it must use near-field beam-forming method. In the far-field case, it is usually used for the far-field acoustic source azimuths estimate. But it is different in near-field conditions because the angles and distances between different sensors on the same array and the point source (i.e. the focused acoustic source) are different, we need to estimate the angle and distance between the point source and a sensor. The beam-forming schematic diagram of near-field of linear array is indicated as Figure 1, and $\mathrm{M}$ is the element number.

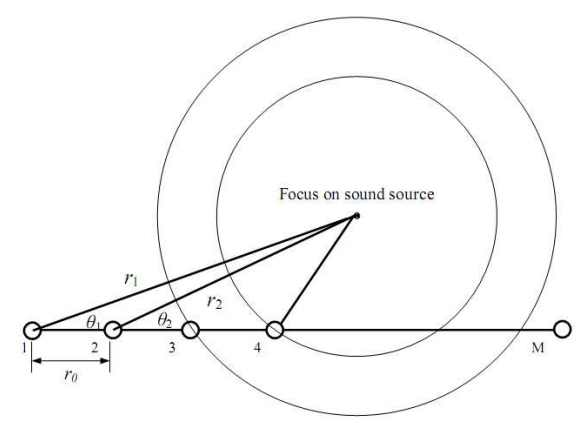

Fig. 1: Near-field beam-forming schematic diagram of linear array

Select sensor 1 as the reference point in this model and assume that the distance between the focusing acoustic source point and the reference point is $\mathrm{r} 1$, the azimuth is 1 . Then the distance difference between the focusing acoustic source point to the ith sensor and to sensor 1 is:

$$
\Delta r_{i}=r_{1}-\sqrt{r_{1}^{2}+(i r)^{2}-2 i r r_{1} \cos \theta_{1}} i=2,3, \cdots M
$$

Where $r_{1}$ in the above equation stands for the distance between the focused acoustic source point and sensor 1, and $r$ stands for the distance between two adjacent array elements of linexar array. Set the propagation speed of acoustic emission in the material as c, the time delays of the received signals of each sensor relative to the reference point are:

$\tau_{i}\left(\theta_{1}, r_{1}\right)=\frac{r_{1}-\sqrt{r_{1}^{2}+(i r)^{2}-2 i r r_{1} \cos \theta_{1}}}{c} i=2,3, \cdots M$
Then, the beam-forming algorithm based on time-domain weighted summation is:

$$
b\left(\theta_{1}, r_{1}, t\right)=\sum_{i=1}^{M} w_{i} A\left(r_{i}\right) x_{i}\left(t-\tau_{i}\left(\theta_{1}, r_{1}\right)\right)
$$

The $x_{i}$ in the above equation stands for the signal measured by the ith array element, $w_{i}$ stands for the ith array element weighting coefficient, $w_{i}=e^{-j 2 \pi f \tau_{i}\left(\theta_{1}, r_{1}\right)}$, in this equation, $f$ stands for signal frequency. $\tau_{i}\left(\theta_{1}, r_{1}\right)$ stands for the time delay of the ith sensor with respect to the reference point when it is focused to some point. For the target source, we can adjust the signals to the same wavefront via $\tau_{i}\left(\theta_{1}, r_{1}\right)$ before the addition of signals i.e. there shall be a cophasal superposition of signals after the time delay compensation, thus beam output in this direction is the largest and the beam output will becomes correspondingly smaller when focused on other points which plays a spatial filtering role. $A\left(r_{i}\right)$ stands for the energy attenuation function of acoustic emission signals on the test rig guided-wave plate, this function is not applied only to a specific frequency or a certain wave mode, but a wave group. Method of calculation is: set a linear sensor array of equidistance on the guided-wave plate, the first point of the array is the rubbing source which will be used to simulate the first rubbing acoustic emission event, collect acoustic emission signals from each array element respectively and calculate the total acoustic emission energy of all array elements in the same period of time, we can get the energy attenuation ratio of the rubbing source and each point of different distance, then the energy attenuation function will be got by curve fitting. The significance of Eq. (3) lies in: the energy spectrum concentrated on some points can be obtained by time delaying, weighting and summing operations on the output of each array element. If a focus point overlaps the target source, adjust the output signals of array elements right to the same wave via time delay compensation on each element i.e. the focus point signals reach the array with the same phase so that it will generate a maximum value of response in the energy spectrum forming a major lobe, the direction of the major lobe is the direction of the target source. The above algorithm assumes that the source signal is of a single frequency and ignores the noise influence in the weighted summation process. In fact, rubbing acoustic emission source signals are the wave packet including large number of frequency components mixed with noises during the propagation of signals. Under such circumstances, it means that there are many source signals with different frequencies in the space, the signal received by the sensor is the superposition of many source signals. Therefore, it is difficult to achieve the separate location of source signals only in time domain. We can also implement frequency domain processing on array signals to get the beam-forming algorithm based on frequency domain. For the linear array with $\mathrm{M}$ array elements of equidistance, 
each element receives $\mathrm{N}$ data within the time of $T_{0}$, carry out Fourier transformation on these data of $M$ groups to get the spectrum of received signals of each array element:

$$
\begin{aligned}
& X_{i}(\omega)=\sum_{n=0}^{N-1} x_{i}(n) W_{N}^{n \omega}=\sum_{n=0}^{N-1} x_{i}(n) e^{-j \frac{2 \pi}{N} n \omega}, \\
& i=1 \cdots M
\end{aligned}
$$

The weighted output of beam-forming algorithm in frequency domain is as follows:

$$
B(\mathbf{r}, \omega)=\sum_{i=1}^{M} w_{i} X_{i}(\omega) e^{-j \omega \tau_{i}(\mathbf{r})}
$$

Where $r$ is a vector and its size equals the distance between the focused point and the sensor; the direction of the vector is the angle between the focal point and the reference array element. Substitute Eq. (4) to (5) and simplify it then we can get the normalized frequency domain beam-forming output:

$$
B(\mathbf{r})=\frac{1}{M} \sum_{i=1}^{M} w_{i} e^{\frac{-j 2 \pi \omega(i-1) \tau_{i}(\mathbf{r})}{N}}\left(\sum_{n=0}^{N-1} x_{i}(n) e^{\frac{-j 2 \pi k n}{N}}\right)
$$

\section{Simulation analysis of rubbing acoustic emission source location based on near-field beam-forming}

Set the acoustic source signal frequency to $1,000 \mathrm{~Hz}$, the propagation velocity of the acoustic source to $1,500 \mathrm{~m} / \mathrm{s}$ and the signal azimuth with respect to the reference point to $40^{\circ}$. Besides, set a directional interference signal with a frequency of $5,000 \mathrm{~Hz}$, a propagation velocity of $3,000 \mathrm{~m} / \mathrm{s}$ and equal amplitude with the acoustic source signal in the place of $10^{\circ}$ direction angle with respect to reference point. The signal sampling frequency of this system is $15 \mathrm{kHz}$, add a white Gaussian noise with SNR of $10 \mathrm{~dB}$ which is not directional and belongs to a kind of background noises to the output of each array element. Set the array element spacing to $18 \mathrm{~cm}$, element number to 32 , its formation is linear array, and its azimuth angle can be searched in the range from $-90^{\circ}$ to $90^{\circ}$ evenly. The calculation results of time domain and frequency domain beam forming are separately shown in Figure 2 and Figure 3.

As can be seen from Figure 2, for the directional interference, time-domain beam-forming can commendably distinguish the azimuth angle between the interference and the signal. In Figure 3(a), both signal and interference produce a local peak in the frequency domain beam-forming diagram which not only points out the definite azimuth angles of signal and interference, but also gets the specific frequency band to which they are located.

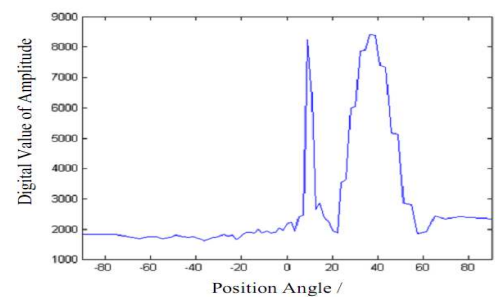

Fig. 2: Formation diagram of time-domain beam with 32 array elements

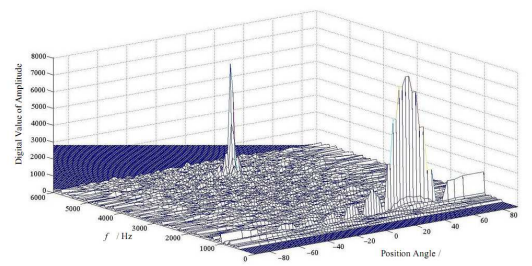

(a)Three-dimensional image of frequency-domain beam-forming

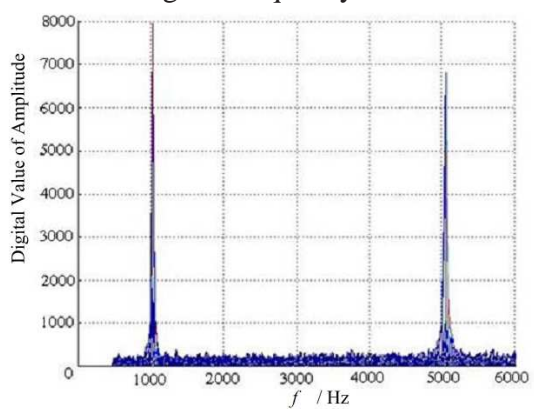

(b) Y-Z direction view of frequency-domain beam-forming

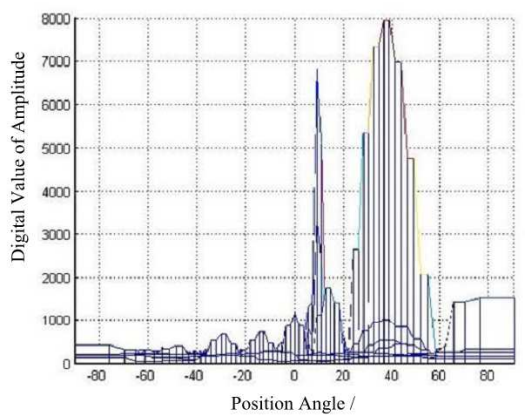

(c) $\mathrm{X}-\mathrm{Z}$ direction view of frequency-domain beam-forming

Fig. 3: Formation diagram of frequency-domain beam with 32 array elements

Simulation experiments show that both the time-domain and frequency-domain beam-forming algorithm are estimates of the distance and direction of the focal point, the time-domain algorithm shall identify the acoustic source point once according to the entire band, when there is interference in space which is different from the location of the source point, the beam pointing diagram 
will form a peak in these two locations at the same time, and when the energy of the interfering signal is relatively strong, the peak energy it produced will be greater than that of the acoustic source point which may lead to the misjudgment of the real source point. Comparatively speaking, frequency-domain algorithm can get a higher estimation accuracy, we can make use of the prior knowledge to get an approximate actual acoustic source signal band, and when the background noise is strong, frequency domain beam-forming method can select local band of the signal frequency for energy weight to effectively shield the impact of noise on other bands and ultimately improves the accuracy of recognition location. Frequency-domain beam-forming method increases the amount of computation compared with time-domain method because the computation needs to be converted to the frequency domain.

\section{Forming of near-field MVDR beam}

Traditional near-field beam forming method is established on the basis of accurate data, while in practical applications, many factors, including less beat number due to the fast speed, inaccurate estimate of signal arrival angle, uneven signal propagation medium, concentration of excessive signal energy on low frequency band and so on, would seriously affect the performance of beam-forming algorithm, especially the restriction of the sensor's installation condition on rotating machinery. Moreover, the number of sensor arrangement is limited which makes the number of array elements is small, and it will result in a serious decline of the azimuth resolution rate. MVDR is a direction estimation method with high resolution rate, and its basic idea is that the expected direction signal will pass through without distortion and with a certain gain which minimizes the interference and noise power. Since the given position spectrum by the algorithm uses all the eigenvalues of data sampling covariance matrix, among which the signal characteristic value which plays a leading role reflects the power of the acoustic source and the relative size of the source contribution. Therefore, it reduces the contribution of interference signal and noise while retaining the useful signal, obtains the higher location resolution and noise suppression performance, and it has more advantages over the traditional beam-forming method in near-field acoustic source location.

\subsection{Forming of near-field MVDR beam}

Assume that the signal frequency is $f_{0}$, array element spacing is $r$, wave velocity is $c$, and it evenly distributes $\mathrm{M}$ array elements in the linear array, and under far-field circumstance, the signal shoots on the linear array with an incidence angle of $\theta_{0}$, the covariance matrix $R_{x}$ of $X(t)$ is as follows:

$$
R_{X}=E\left[X(t) X^{H}(t)\right]=A R_{S} A^{H}+\sigma^{2} I
$$

Here, $X_{H}(t)$ is the conjugate transpose of $X(t), R_{S}$ equals $E\left[S(t) S_{H}(t)\right]$ i.e. the correlation matrix of the source signal among the received signals, and $\delta^{2}$ stands for the noise power, I stands for an M-order identity matrix. $w_{i}$ is chosen as the weighted value of the ith array element signal $x_{i}(t)$, then the output of the traditional beam-former is as follows:

$$
Y_{C B F}(t)=W^{H} X(t) \sqrt{2}
$$

where $\mathrm{W}=\left[\mathrm{w}_{1}, \mathrm{w}_{2}, \ldots \mathrm{w}_{\mathrm{M}}\right]^{\mathrm{T}}$ in the above equation stands for the weight vector matrix, and $X(t)$ stands for the input matrix of array element. Then the array output power is as follows:

$$
P_{C B F}(t)=E\left\{|y(t)|^{2}\right\}=W^{H} R_{X} W
$$

The core idea of the MVDR algorithm is to keep the signal energy of the incoming wave direction unchanged and minimum the signal energy on other directions, so it is essentially a constrained optimization problem. For the traditional beam-forming algorithm, the weight vector matrix $W_{C B F}$ is constant, but in the MVDR algorithm, WMVDR shall be adaptively given by the actual received signal ambient noise and strength and distribution of the real space target $[16,18]$.

$$
\min \left\{W_{M V D R}^{H} R_{X} W_{M V D R}\right\} \text { subject to } W^{H} \mathbf{a}(\theta)=1
$$

Here, $a(\theta)$ stands for the direction vector of $\theta$ direction, and $a(\theta)$ equals $\left[1 e^{-j 2 \pi f d \sin \theta / c} \ldots e^{-j(M-1) 2 \pi f d \sin \theta / c}\right]$. The purpose of constraint that $W_{H} a(\theta)$ equals 1 is that the response of the fixed weight vector $\mathrm{W}$ in the viewing direction $\theta$ is the conventional coherent summation so that the contribution of noise and interference not in the direction is the minimum. The optimal solution of Eq. (10) is as follows:

$$
W_{M V D R}=\frac{\left(R_{X}^{*}\right)^{-1} \mathbf{a}^{*}(\theta)}{\mathbf{a}^{H}(\theta) R_{X}^{-1} \mathbf{a}(\theta)}
$$

So the spatial spectrum of MVDR algorithm in far-field conditions is as follows:

$$
P_{M V D R}=\frac{1}{\mathbf{a}^{H}(\theta) R_{X}^{-1} \mathbf{a}(\theta)}
$$

Here, $a H(\theta)$ is the conjugate transpose operation of matrix $a(\theta)$, and $a^{*}(\theta)$ and $R_{X}^{*}$ separately stands for the conjugate operation of matrix $a(\theta)$ and $R_{X}$. In an ideal situation, the space spectrum of $M_{V D R}$ algorithm is approximately a function. It's noticed that both $W_{V D R}$ and $P_{V D R}$ in Eq. (11) and (12) are relevant to the correlation matrix RX of the received signals, so the precision of $M_{V D R}$ is influenced by the SNR. The above-mentioned 
$M_{V D R}$ methods are deduced according to the far-field assumption which does not have the distance resolution capability i.e. it can not measure the distance or distinguish the acoustic source of angles in the same direction. Under near-field circumstance, $M_{V D R}$ algorithm brings in the impact compensation of distance on the time delay, so it can estimate the distance and azimuth angle simultaneously. Assume that the distance between the scan point and the far left element (its number is 1) is $r_{1}$ and the azimuth angle at this point is $\theta_{1}$, so the distance between the scan point and the rest elements can be expressed by $r_{1}$ and $\theta_{1}$. The source point location can be determined if we can estimate the values of $r_{1}$ and $\theta_{1}$.

$$
r_{i}=\sqrt{r_{1}^{2}+(r i)^{2}-2 r_{1} r i \cos \theta_{1}} i=2 \cdots M
$$

Then the direction vector a is the joint two-dimensional function of $r_{1}$ and $\theta_{1}$ :

$$
\mathbf{a}(r, \theta)=\left[1, e^{-j 2 \pi f\left(r_{1}-r_{2}\right) / c} \cdots e^{-j 2 \pi f\left(r_{1}-r_{M}\right) / c}\right]
$$

Accordingly, under the condition of near-field, the spatial spectrum of $M_{V D R}$ algorithm is as follows:

$$
P_{M V D R}=\frac{1}{\mathbf{a}^{H}(r, \theta) R_{X}^{-1} \mathbf{a}(r, \theta)}
$$

Scan every point in the sound field and we can get the two-dimensional joint distribution function of array output power in respect to $r$ and $\theta$. When the array scanning point and the actual location of the acoustic source is coincident, the output power of cophasal superposition produced by the signal is the biggest one and a peak will be formed in the acoustic diagram. The spatial resolution of $M_{V D R}$ algorithm is influenced by many factors such as the signal frequency, the number of array elements, array element spacing and the distance between the actual acoustic source point and the array. The higher the frequency is, the greater the array size is and the closer the acoustic source apart from the array, the higher the resolution will be.

\subsection{The wideband signal MVDR location estimation based on sub-band decomposition}

The above algorithm is designed for narrow-band signals. For narrowband signals, the shifting phase method is usually used, while the incident signal is a wideband one, we can not use the traditional phase-shift method to form the correct beam direction because different frequencies correspond to different phase shift values. As for such wideband signals as rubbing acoustic emission signal, if we simply apply the above algorithm, it may lead to large errors and even incorrect results. Wideband signal can be seen as the summation of a number of narrowband signals of adjacent frequency. There are two directions of wideband signal processing, one is irrelevant signal-based processing method i.e. decompose the wideband data into narrowband data which is on non-overlapping frequency bands, then process and estimate the initial angle each narrow-band, and at last, we can get the final result according to the combination of these initial estimates. The other one is coherent signal-based processing method i.e. focus on the signal space of the non-overlapping frequency points on the band to the reference frequency points, then focus on the wideband signals to the reference frequency points by the focusing matrix, and finally estimate the direction by the narrowband signal processing method. Irrelevant signal processing method needs to perform a position estimate on every frequency, so there is a large amount of computation; and the key of the algorithm is to calculate the focusing matrix, the matrix dimension will be very large when the number of sampling points is large, so it will be very difficult to focus all the signals on a particular frequency by the focusing matrix. Rubbing acoustic emission signal has a very large amount of data, for example, when the rotational speed is $600 \mathrm{r} / \mathrm{min}$ and the sampling frequency is $500 \mathrm{KHz}$, the number of sampling points of a rubbing cycle will be up to $50 \mathrm{~K}$ bytes, and there will be a very large amount of computation if the focus transformation is performed on these data, and this algorithm will not be suitable for practical applications. In this paper, we will divide the $M$ group received wideband signals into $S$ segments in the time domain, the length of each segment is $N_{0}$ points and do fast Fourier transform on each segment of data to form a band with $f_{0} / 2$ bandwidth, thus frequency snapshots will be formed in each sub-band.

$$
X\left(n, f_{k}\right)=\left[X_{1}\left(n, f_{k}\right), X_{2}\left(n, f_{k}\right) \cdots X_{M}\left(n, f_{k}\right)\right]
$$

$X_{i}\left(n, f_{k}\right)$ stands for the frequency component of the nth frequency snapshot of the ith group signal of the based array with the frequency of $f_{k}$, and $X_{i}\left(n, f_{k}\right)$ stands for the frequency component of all the $n$-th snapshots of the signal with the frequency of $f_{k}$, so the covariance matrix under this frequency is as follows:

$$
\tilde{R}_{x}\left(f_{k}\right)=\frac{1}{U} \sum_{n=1}^{U} X\left(n, f_{k}\right) X^{H}\left(n, f_{k}\right)
$$

The beam output power when the direction angle of this frequency component is $\theta$ is as follows:

$$
P_{M V D R}\left(r, \theta, f_{k}\right)=\frac{1}{a^{H}\left(r, \theta, f_{k}\right) \tilde{R}_{x}^{-1}\left(f_{k}\right) a\left(r, \theta, f_{k}\right)}
$$

The direction vector $\mathbf{a}\left(r, \theta, f_{k}\right)$ in the above equation equals $\left[1, e^{-j 2 \pi f_{k}\left(r-r_{2}\right) / c} \cdots e^{-j 2 \pi f_{k}\left(r-r_{M}\right) / c}\right]$. Add up all the narrow-band powers and normalize it, then we can get the wideband output power:

$$
P_{M V D R}(r, \theta)=\frac{1}{N_{f}} \sum_{k=1}^{N_{f}} P_{M V D R}\left(r, \theta, f_{k}\right)
$$


Where $N_{f}$ stands for the number of sub-band decomposition, and after the FFT operation, we can get the effective signal frequency range i.e. from 0 to $f_{0} / 2$. The frequency resolution of the frequency spectrum $\Delta f$ equals $f_{0} / N_{0}$, For example, when $N_{0}=f_{0} / 2$, the frequency resolution is $2 \mathrm{~Hz}$, then after the FFT operation, there are only $N_{0} / 2$ points in the arrange from 0 to $f_{0} / 2$, so $\mathrm{Nf}$ equals $N_{0} / 2$ i.e. it will weights once every other $2 \mathrm{~Hz}$. When the array scans to the acoustic source point i.e. $r \rightarrow r 1$ and $\theta \rightarrow \theta_{1}, P_{M V D R}$ will form a peak. Since the MVDR algorithm weights on the entire frequency domain and it combines the azimuth spectrum of all bands to get the wideband estimated result which makes its noise immunity stronger than that of the conventional beam-forming algorithm. This algorithm can also effectively make the distinction in space domain especially for directional noise or interference. At the same time, the adoption of sub-band decomposition method makes it possible that MVDR algorithm can avoid the harsh requirement that the signal must be narrowband, so it can be extended to the application in the wideband signal. The flow chart of the algorithm is shown in Figure 4. Calculation steps of the algorithm are as follows:

(1) Collect rubbing AE signals. If the number of array elements is M, M groups of data shall be collected. Rubbing AE signals belong to wideband signals.

(2) Sub-segment decomposition. Divided the received $\mathrm{M}$ groups of wideband acoustic emission signals into several segments in time domain.

(3) Carry out FFT operations on each sub-band.

(4) Calculate the covariance matrix and the direction vector in each frequency.

(5) Calculate the MVDR power output of each subband.

(6) Synthesize MVDR power of wideband signals.

(7) Scan all points in the acoustic field, the synthesized MVDR power will form a peak in the spatial spectrum when the array scans to the acoustic source point, space between the corresponding acoustic source of this peak value and the reference point and incidence angle consist the location coordinates of rubbing acoustic emission source point.

Add directional noise data to the data collected in the step (1), thus it can be used to simulate rubbing AE signals containing noise.

\section{Experiment analysis}

Figure 5(a) shows the rotor rubbing test rig which is a rotor system containing three bearings and two cross components, these three bearings are sliding bearings which can simulate rotor imbalance shaft system misalignment, rubbing and other faults. Rubbing of rotor-stator of the rotor system can be simulated through rubbing bracket which is shown in Figure 5 (b). Install the bracket between bearing 2 and bearing 3 , it can be moved and fixed on the rotor rig base, implement firm coupling

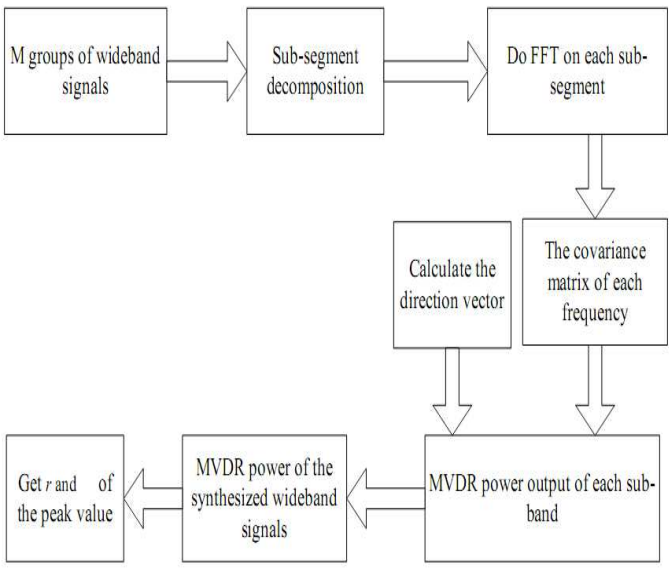

Fig. 4: Wideband signal MVDR algorithm flow diagram based on sub-band decomposition

connection between the rubbing screw and the guided-wave plate, adjust the bracket to make the rubbing screw located in the side of the rotor plate facing the shaft center along the shaft radial direction, then rubbing faults of different degrees can be simulated by adjusting the rubbing screw. AE signals generated by rubbing source can be coupled to the guided-wave plate through rubbing screw and then propagated to AE sensor array. The position of rubbing screw here is the focused point acoustic source; its propagation diagram is shown in Figure 1. The experiment selects UT-1000 sensor with the frequency response ranging from $60 \mathrm{kHz}$ to $1000 \mathrm{kHz}$. The gain of preamplifier is $40 \mathrm{~dB}$ and the $\mathrm{A} / \mathrm{D}$ resolution is 18 bit. Most rotary mechanical rubbing faults are always shown as local rubbing which periodically generates a cluster of high-energy acoustic emission signals, and the energy between the adjacent two clusters of rubbing acoustic emission signals is much smaller and it is mainly caused by mechanical noise, environmental noise and electromagnetic noise. A large number of experiments show that the energy of rubbing acoustic emission signal is concentrated on frequency segments below 100k. Figure 6 shows the time domain and frequency domain diagrams of the continuous rubbing acoustic emission signal when the rotational speed is $350 \mathrm{r} / \mathrm{min}$ and the sampling frequency is $500 \mathrm{kHz}$; Figure 7 (a) is a wave cluster of Figure 6, and Figure 7 (b) shows the signal details of a shorter time period. It can be seen that all the generated rubbing acoustic emission frequency spectrums are approximately the same whose energy are mainly concentrated on the frequency segment from $20 \mathrm{kHz}$ to $50 \mathrm{kHz}$. In order to reduce the computation, it only needs to weight the frequency segment from $20 \mathrm{kHz}$ to $100 \mathrm{kHz}$ after the Fourier transform of each sub-segment, and the signal energy of the rest frequency bands can be considered to be zero. 


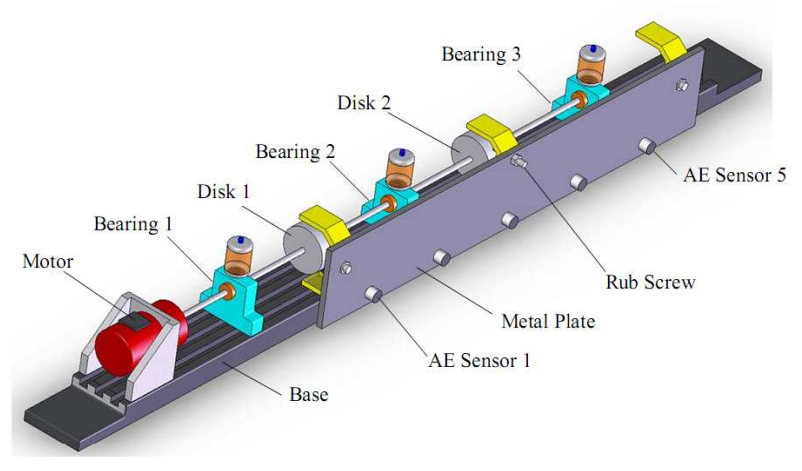

(a)Rotor rubbing test rig

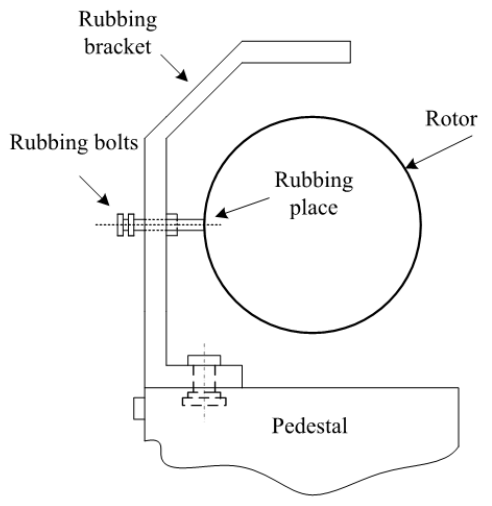

(b) Rubbing bracket

Fig. 5: Rubbing test equipment diagram
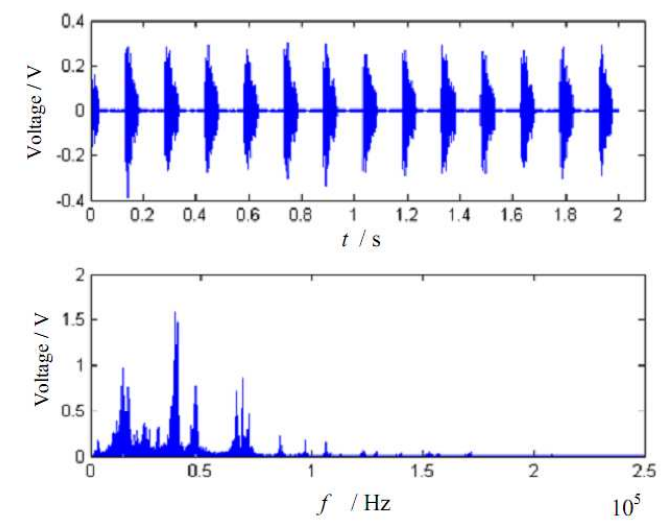

Fig. 6: Time and frequency diagram of continuous rubbing acoustic emission signal

\subsection{Location simulation of wideband signal MVDR based on sub-band decomposition under different conditions}

Set that the acoustic source signal frequency ranges from $20 \mathrm{kHz}$ to $100 \mathrm{kHz}$, the acoustic velocity is $1,500 \mathrm{~m} / \mathrm{s}$, the
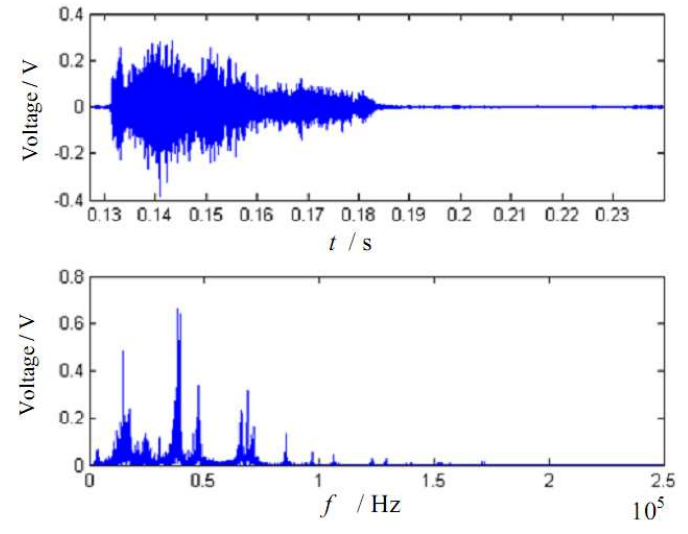

(a)Partial diagram of rubbing acoustic emission signals
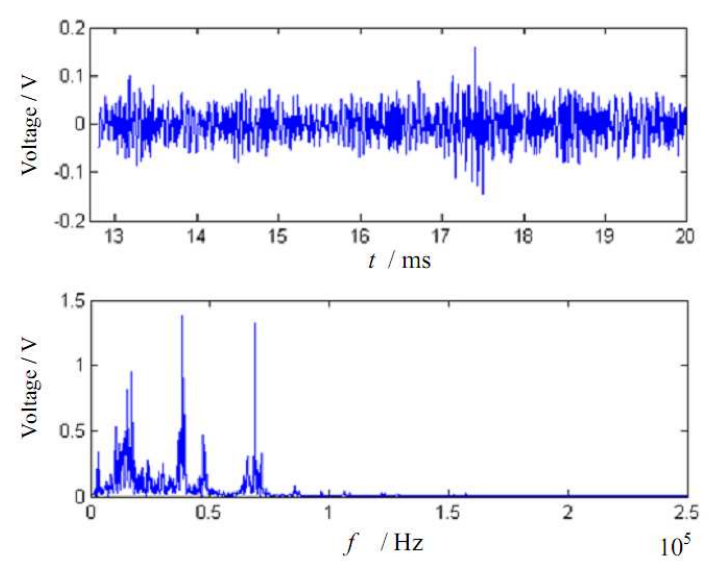

(b) Signal commenced details with time length of $7 \mathrm{~ms}$

Fig. 7: Time and frequency diagram a cluster of rubbing acoustic emission signal

sampling frequency is $200 \mathrm{kHz}$; the number of snapshots is 20 and the formation is linear array. The azimuth angle between the acoustic source and sensor 1 is $60^{\circ}$, the distance $r$ is $4.50 \mathrm{~m}$; the array element spacing is $0.2 \mathrm{~m}$. stack white noise of different SNR to the collected acoustic emission signals. Divide the target signal frequency band into sub-bands every $100 \mathrm{~Hz}$, considering that the bandwidth of acoustic source signal is $80 \mathrm{kHz}$, totally 801 frequency points will be just enough to cover the entire frequency band. Calculate the beam energy of each frequency point and put them into Eq. (19) to be summed up, then we can get the MVDR spatial spectrum function about distance and azimuth angle. Figure 8 shows the calculation result of wideband near-field signals MVDR method based on sub-band decomposition of different SNRs and different array numbers. It can be seen that this method can correctly estimate the azimuth angle and the distance of the target signal under near-field wideband circumstance, the larger the number of array elements is and the higher the SNR is, the sharper the 


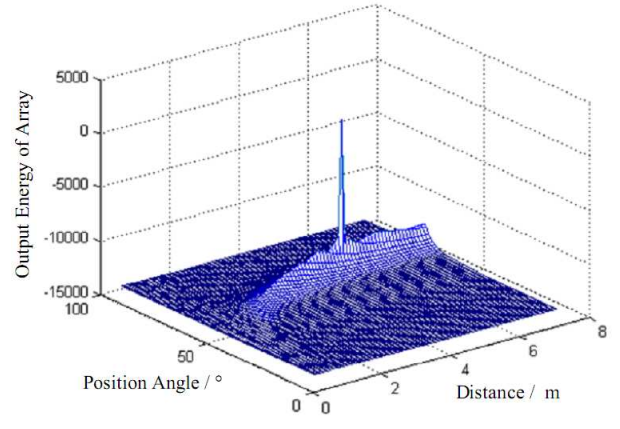

(a)SNR is $10 \mathrm{~dB}$, the number of array elements is 5

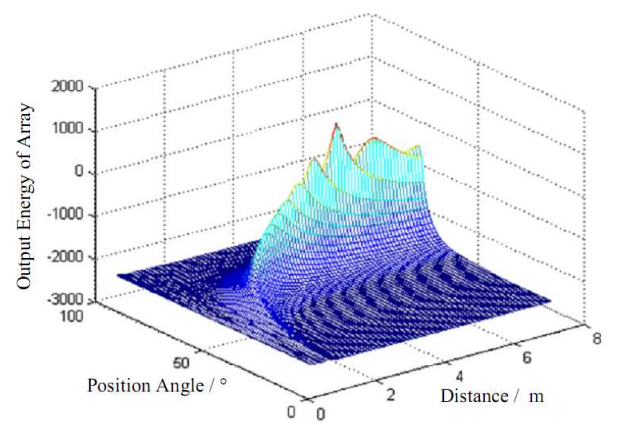

(b) SNR is $5 \mathrm{~dB}$, the number of array elements is 5

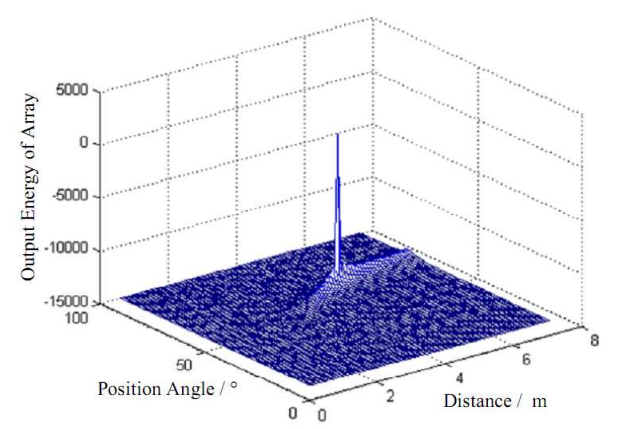

(c)SNR is $10 \mathrm{~dB}$, the number of array elements is 15

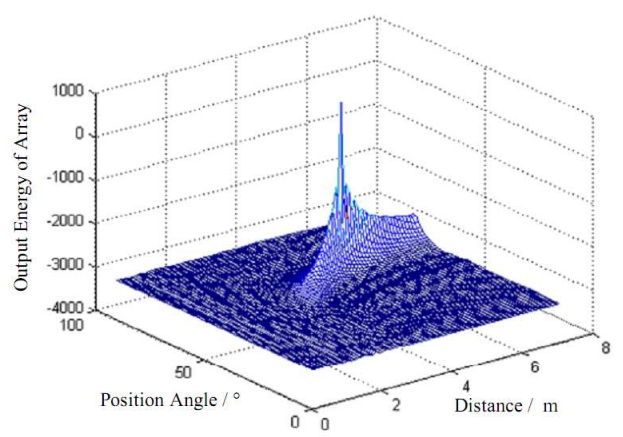

(d)SNR is $5 \mathrm{~dB}$, the number of array elements is 15

Fig. 8: Wideband signal MVDR azimuth estimates based on sub-band decomposition of different SNR and different array elements number

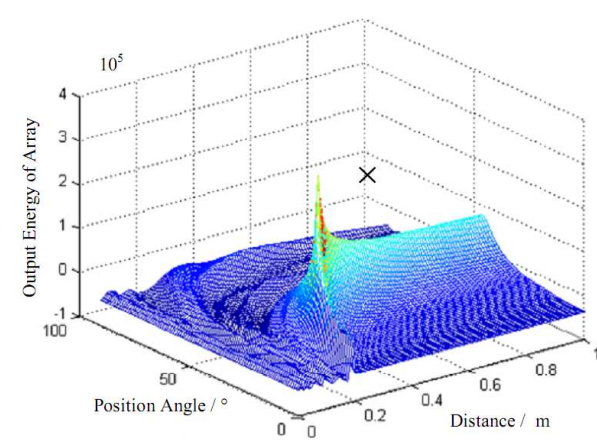

(a)emission signals without noise

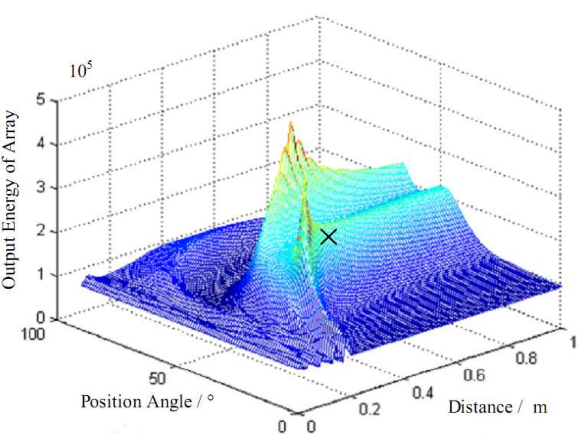

(b)emission signals with noise

Fig. 9: The direction estimation diagrams of rubbing acoustic emission signal MVDR

main lobe will be and the lower side lobes' energy will become. At the same time, it can also be seen from Figure 8 that the algorithm will form a number of "false peaks" in other positions which approximately is a hyperbola in the polar diagram. This is because that there are numerous points of a constant distance difference to the two array elements in the plane, these points form a hyperbola, the more the passing hyperbola of a point there are, the greater its output will be, and the intersection point of all hyperbola is the main lobe peak value. It will form a maximum peak value in all the hyperbola intersections when the number of array elements is relatively small, but it will form a relatively large peak value in other intersections which can not make the main lobe more obvious from the side lobes. This is the reason why the peak value in Figure 8 (d) is more obvious than that in Figure 8 (b) when the SNR are the same.

\subsection{Actual rubbing acoustic emission source location}

Take the actual rubbing acoustic emission signal in Figure 6 as the source signal, assume that the distance between the rubbing source (the position of rubbing screw on 
guided-wave plate) and sensor 1 is $300 \mathrm{~mm}$, the azimuth angle is $30^{\circ}$, the array element spacing is $50 \mathrm{~mm}$, the element number is 5 , the array formation is linear array and sensor 1 is the reference point of the array. Assume that there is a white Gaussian noise whose energy level is the same as the source signal in the position with an azimuth angle of $60^{\circ}$ and its distance to the sensor is $500 \mathrm{~mm}$. After calculation, the rubbing acoustic emission wave propagation velocity in the guided-wave plate is about $3,000 \mathrm{~m} / \mathrm{s}$, and the received signals of each element come after the time delay and stacking process of the source signal and the noise signal. The received signals of each array element are divided into nine snapshots with a length of 50,000 points, so there are totally 5,000 sub-bands in the frequency arrangement from $0 \mathrm{~Hz}$ to $100 \mathrm{kHz}$ with a band-width of $20 \mathrm{~Hz}$, the search angle range is from $0^{\circ}$ to $90^{\circ}$ and the distance range is from $0 \mathrm{~mm}$ to $1000 \mathrm{~mm}$.

Figure 9 (a) shows the MVDR spatial spectrum of rubbing acoustic emission signal after sub-band decomposition, and we can see that MVDR space spectral estimation based on sub-band decomposition forms a peak value in the rubbing source, and the peak value point is located on the position with a distance of $300 \mathrm{~mm}$ and a azimuth angle of about $30^{\circ}$. Figure 9 (b) shows the spatial spectrum of rubbing acoustic emission signal MVDR with noise, and we can see from the diagram that it forms a peak separately in the rubbing source and the noise source which are very close to the actual location. It is different from the frequency domain beam-forming method which distinguishes the signal from the noise in terms of frequency; MVDR beam-forming method based on sub-band decomposition distinguishes the signal from the noise in terms of space position. If we have some prior understanding of the acoustic source position before the calculation, we can also narrow the search area and reduce the search step size to further improve the accuracy.

\section{Conclusion}

In this paper, the beam-forming method is applied to the rotating machinery rubbing acoustic emission source location, and simulation study is performed on the performance of traditional near-field beam-forming in rubbing acoustic emission source location and the wideband MVDR location estimation method based on sub-band decomposition is adopted. The conclusions are as follows:

(1) In case of large number of array elements, both time domain and frequency domain beam-forming can effectively distinguish the location of the interference and the signal. But when the interference signal is relatively strong, the formed peak energy of it will be greater than that of the acoustic source, so the time-domain method might produce misjudgment on the real source position. If we can get the approximate frequency band of the actual acoustic source signal by the priori knowledge, we can select the local frequency band where the signal frequency locates and weight the energy of it by the frequency domain wave method, and finally we can effectively shield the influence of noise on other frequency bands and get a better positioning accuracy.

(2) When there are relatively few array elements, the wideband signal MVDR spatial spectral estimation based on sub-band decomposition can efficiently distinguish the signal and the noise in the aspect of space, and accurately locate the rubbing source.

(3) Beam-forming is able to effectively diagnose the rubbing acoustic emission source location which provides a new way for rubbing fault source location.

\section{Acknowledgement}

This work was supported by the Natural Science Foundation of China under Grant No.51075068, No. 60872073, No.60975017 and the Foundation of Huaqiao University (No.12BS228).

\section{References}

[1] Sato I, Rotating machinery diagnosiswith acoustic emission techniques Electr Engng Jpn, 110, 115-127 (1990).

[2] Suzuki H, T Kinjo, Y Hayashi,et al, Wavelet transform of acoustic emission signalsJAcoustic Emission, 14, 69-84 (1996).

[3] Mba D., Hall L. D., The transmission of acoustic emission across large scale turbine rotorsNDT and E International, 35, 529-539 (2002).

[4] Hall L. D. , Mba, D., Diagnosis of continuous rotor-stator rubbing in large scale turbine units using acoustic emissions. Ultrasonics, 41, 765-773 (2004).

[5] Omologo M, Svaizer P, Acoustic source location in noisy and reverberant environment using CSP analysisProcessing of ICASSP, 921-924 (1996).

[6] Yegananarayana B, Mahadeva P S, Ramani D, et al Processing of reverberant speech for time-delay estimationIEEE Transaction on Speech and Audio Processing, 13, 1110-1118 (2005).

[7] Deng Aidong, Bao Yongqiang, Zhao Li Research on time delay estimation algorithm based on generalized cross correlation in acoustic emission source locationProceedings of the CSEE, 29, 86-92 (2009).

[8] Ciampa, Francesco, Meo, Michele. Acoustic emission source localization and velocity determination of the fundamental mode A0 using wavelet analysis and a Newton-based optimization technique. Smart Materials and Structures, 19, (2010).

[9] Dirk Aljets, Alex Chong, Steve Wilcox, Karen Holford. Acoustic emission source location on large plate-like structures using a local triangular sensor array. Mechanical Systems and Signal Processing, 30, 91-102 (2012). 
[10] Aidong Deng, Li Zhao, Xin Wei The Application of Wavelet Neural Network Optimized by Particle Swarm in Localization of Acoustic Emission SourceProceedings of 16th International Conference on Neural Information Processing,ICONIP, 738-745 (2009)

[11] Van Veen B D, Buckley K M. Beamforming:A versatile approach to spatial filtering. IEEE ASSP Magazine, 5, 4-24 (1988).

[12] Koh C L. A comparison of adaptive beamforming implementations for wideband scenarios. 2nd IEE EURASIP Conference On DSP enabled Radio. London: IEE Press, 9 - 13 (2005).

[13] McLaskey, Gregory C.,Glaser, Steven D, Grosse, Christian U. Beamforming array techniques for acoustic emission monitoring of large concrete structures. Journal of Sound and Vibration, 329, 2384-2394 (2010).

[14] 14. He Tian, Pan Qiang, Liu Yaoguang. Near-field beamforming analysis for acoustic emission source localization. ULTRASONICS, 52, 587-592 (2012).

[15] Lorenz R G, Boyd S P. Robust minimum variance beamforming. IEEE Trans. Signal Processing, 53, 16841696 (2005).

[16] iang Biao , Zhu Ye , Sun Changyu, et al. Research on an efficient wideband high resolution MVDR algorithm. Systems Engineering and Electronics, 27, 1186-1188 (2005).

[17] He Xinyi, Jiang Xingzhou, Li Qihu. Research on broadband beamspace minimum variance distortionless response high resolution direction-of-arrival estimation method based on sub-band decomposition. Shengxue Xuebao/Acta Acustica, 29, 533-538 (2004).

[18] You Hong, Huang Jianguo, Xu Guimin. Beamspace pre-processing on array data based on MVDR.Systems Engineering and Electronics, 30, 64-67 (2008).

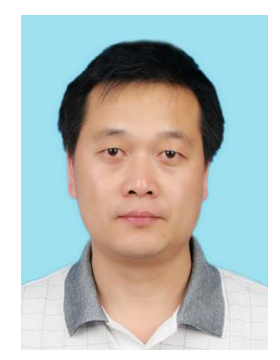

Aidong Deng was born in 1968. He received his B.E. degree in Industrial Electric Automation from Harbin Shipbuilding Engineering Institute in 1991, the M.S. degree in 1994, and the Ph.D. degree in 2008, both from Southeast University. Currently, he is an associate professor with the National Engineering Research Center of Turbo-generator Vibration of School of Energy \& Environment, Southeast University, Nanjing, China. He worked in areas of measurement technology and instruments, fault analyses and signal processing. Recently he has been working on acoustic emission technology for rotating machinery fault diagnosis applications.

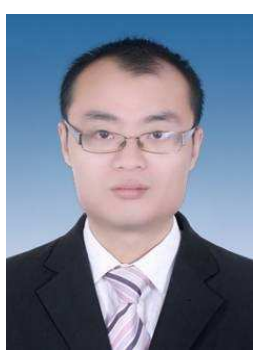

Hang Tong was born in 1987 . He received his B.E. degree from China University of Mining and Technology in 2009 and the M.S. degree from Southeast University in 2012. His research interests include power engineering and fault analyses.

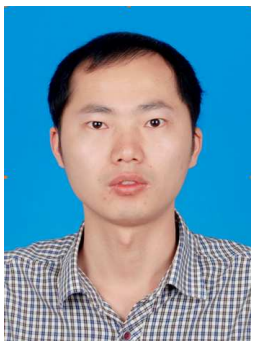

Jianeng Tang was born in 1983. He received his B.E. degree in Electronic Information Science and Technology from Xinjiang Normal University in 2006, the M.S. degree in 2009 from Ningxia University, and the Ph.D. degree in 2012 from Southeast University in Information and

Communication Engineering. Currently, he is with College of Engineering, Huaqiao University, Quanzhou, China. His main research interests include nonlinear systems, chaos synchronization and control, complex networks, acoustic emission technology. 


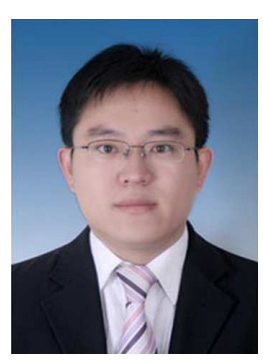

Hao Cao was born in 1982. He received his doctorate from the Department of Power Engineering, Southeast University, in 2012 and is currently working at the Hunan Electric Power Corporation Research Institute in Changsha, China. His research areas are rotating machinery fault monitoring and diagnosis, and rotor dynamics and flow-induced vibration.

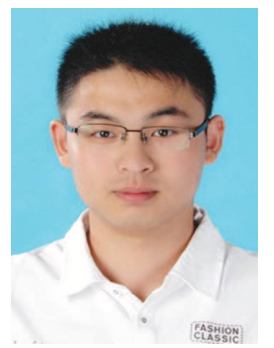

Kang Qin was born in 1988. He received his B.E. degree from Nanjing University of Technology in 2011. Now he is a master degree candidate in Southeast University. His research interests include fault analyses and acoustic emission technology.

\section{Xin}

Yan

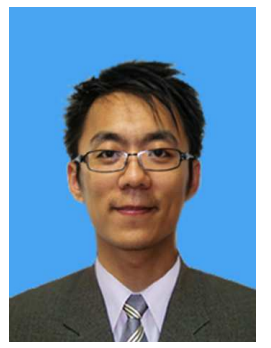

was born in 1989. He received his B.E. degree from Suzhou University in 2011. Now he is a master degree candidate in Southeast University. His research interests include fault analyses and acoustic emission technology. 